\title{
An Isolated Spinal Meningocele Discovered Fortuitously on a CT Scan: Case Report
}

Bouchentouf $\mathbf{R}^{*}$, Benjelloun A and Yasser Z

Pulmonology unit, Avicenna Military Hospital Marrakech, Laboratory PCIM, Cadi Ayyad University, Marrakech, Morocco

\begin{abstract}
A spinal meningocele is a saccular protrusion of the meninges through a dilated intervertebral foramen or a bony defect of the vertebral column. Intrathoracic meningocele is a rare condition. Only a few cases were related in the literature. It is usually associated with neurofibromatosis type 1. Isolated intrathoracic meningoceles without neurofibromatosis are more uncommon. We report a case of isolated paraspinal meningocele in a 54 old-year-man, for whom the diagnosis was established by computed tomography and magnetic resonance imaging features.
\end{abstract}

Keywords: Meningocele; Thoracic; Isolated

\section{Introduction}

Spinal meningocele is a rare disorder defined as a protrusion of the spinal meninges through a defect in the vertebral column or foramina. Only 150 cases of thoracic meningocele have been reported up to 2003 [1]. The authors describe a new case of isolated thoracic meningocele with no associated generalized mesenchymal dysplasia and discuss the differential diagnosis with other conditions such as neurofibromatosis.

\section{Case Report}

A 54 year-old man with a history of pulmonary tuberculosis treated in 1990, with important sequelae, was admitted for mild acute respiratory insufficiency. Physical examination found diminished breath sounds in the right chest, an important cyanosis of the extremities and a 76\% $\mathrm{SaO} 2$ in ambient air. No focal neurologic signs or clinical evidence of neurofibromatosis type 1 (NF-1) was noticed. Laboratory findings were within normal limits. The chest radiograph showed right chest opacity with bronchial distortion evocative of important tuberculosis sequelae.

We concluded to a bronchitis complicating a post-tuberculosis respiratory insufficiency. A respiratory treatment associating oxygenotherapy, steroids, antibiotics and bronchodilators was started. The thoraco-abdominal Computed tomography scan showed a $35 \mathrm{~mm}$ hypodense, round mass with well-defined borders of the left paraspinal region (Figure $1 \mathrm{a}$ and $\mathrm{b}$ ).

Magnetic resonance Imaging MRI showed a cystic mass of the left paraspinal space, communicating with the spinal canal through the

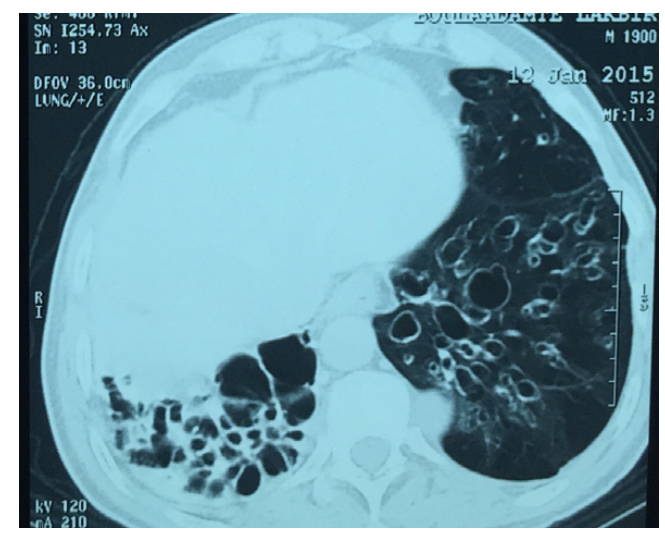

Figure 1: Chest computed tomography scan: $3 \mathrm{~cm}$ hypodense mass of the left paraspinal region.
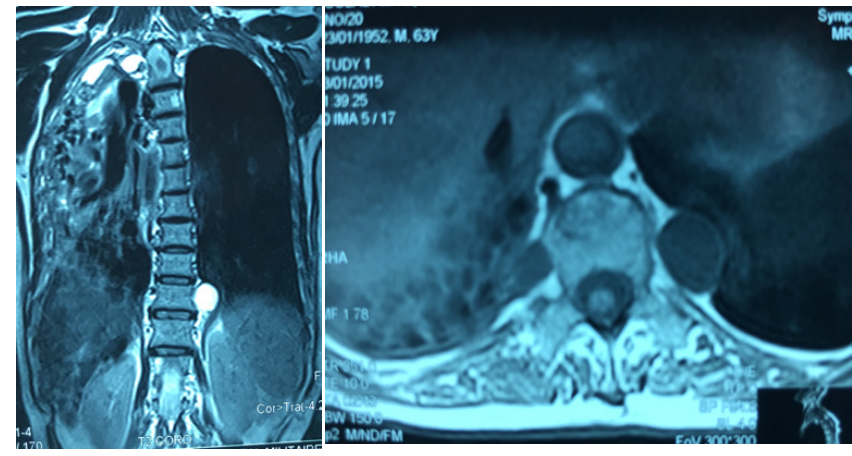

Figure 2: Magnetic Resonance Imaging: cystic mass of the left paraspinal space cerebro-spinal fluid communicating with the spinal canal through the enlarged left intervertebral foramen T 11-12.

enlarged left intervertebral foramen T 11-12 (Figure 2a and b). Given the small size of the lesion and the absence of neurologic signs, a simple follow-up all six month with periodic imaging was recommended without surgical treatment.

\section{Discussion}

The meningocele is a benign and rare lesion defined as a herniation of the meninges through a vertebral column defect. More than $80 \%$ of spinal meningoceles are located in the lumbosacral area. The first intrathoracic location was described in 1933 by Pohl; and has been usually reported as a manifestation of generalized mesenchymal dysplasia such as neurofibromatosis type $1(\mathrm{NF}-1)$ for Marfan syndrome but rarely as an isolated lesion $[1,2]$.

In the thorax, it can be unilateral or bilateral and solitary or multiple. Lateral meningoceles are more frequent because of the relative weakness of the paravertebral muscles and the high pressure gradient between the cerebro-spinal fluid and the thorax [3].

*Corresponding author: Bouchentouf Rachid, Pulmonary unit, Avicenna Military Hospital, 12 Bd la résistance, Marrakech, 40000, Morocco, Tel: +212 5-24-43-48-13; E-mail: bouchentouf_rachid@yahoo.fr

Received May 19, 2015; Accepted June 14, 2015; Published June 16, 2015

Citation: Bouchentouf R, Benjelloun A, Yasser Z (2015) An Isolated Spinal Meningocele Discovered Fortuitously on a CT Scan: Case Report. J Clin Case Rep 5: 545. doi:10.4172/2165-7920.1000545

Copyright: $(2015$ Bouchentouf $\mathrm{R}$, et al. This is an open-access article distributed under the terms of the Creative Commons Attribution License, which permits unrestricted use, distribution, and reproduction in any medium, provided the original author and source are credited. 
Most patients with intrathoracic meningocele are asymptomatic; and incidentally diagnosed on a plain chest $\mathrm{x}$-ray. A few patients present with back pain, cough, or dyspnea due to compression of the lung and the mediastinal structures. The symptoms depend on the size and the location of the meningocele within the thorax. CT scan and/or MRI are necessary to uphold the diagnosis. On CT scan, the meningocele appears as a well-defined, homogeneous, hypodense, paravertebral mass. Plain radiographs can be helpful by showing an enlargement of the spinal canal, posterior vertebral body scalloping, pedicle erosion or a widening of the interpedicular distance. [4].

MRI, when available, is the best examination for this kind of cystic lesions. It usually shows an intradural, extramedullary space-occupying lesion with T1 and T2 weighted signal intensities identical to that of cerebrospinal fluid. The meningocele should be distingushed from posterior mediastinal tumors such as neurofibroma, neuroblastoma, and ganglioneuroma [5]. The difference between isolated meningocele and meningocele associated with neurofibromatosis- 1 is the family history of neurofibromatosis and the presence of café-au-lait spots, cutaneous neurofibromas, and axillary frecklings and Lisch nodules on the iris, and it's associated with osseous lesion including progressive thoracic scoliosis, and vertebral anomalies.

The treatment is a challenge and depends on the size and the complications of meningocele. When intrathoracic meningocele rapidly increases and complaints of pain, neurological or pulmonary signs, and compression of the trachea or esophagus are present, surgical treatment should be considered. It consists on a resection of the meningocele and repair of the dural defect through laminectomy [6]. However, in the case when meningocele was combined with kyphoscoliosis, it is not easy to suture the meningocele through a posterior laminectomy.
In addition, it can bring about instability of the vertebrae after the removal of the meningocele; posterior spinal arthrodesis as well as anterior spinal arthrodesis might be needed [6]. In case of small meningoceles or asymptomatic patients, a regularly follow-up by chest $\mathrm{x}$ ray, Computed Tomography $(\mathrm{CT})$ scans or MRI is indicated.

\section{Conclusion}

The spinal meningocele is a rare condition, often associated with neurofibromatosis type 1 . Most patients with intrathoracic meningocele are asymptomatic; and incidentally diagnosed after a plain chest $\mathrm{x}$-ray. This case report emphasizes that radiological examinations must be analyzed thoroughly to detect asymptomatic lesions which are not always benign.

\section{References}

1. Calzavara PG, Carlino A, Anzola GP, Pasolini MP (1988) Segmental neurofibromatosis. Case report and review of the literature. Neurofibromatosis 1: $318-322$.

2. Jeong JW, Park KY, Yoon SM, Choe du W, Kim CH, et al. (2010) A large intrathoracic meningocele in a patient with neurofibromatosis-1. Korean $\mathrm{J}$ Intern Med 25: 221-223.

3. Mushtaq G, Hussain I, Khan JA, Kamal MA4 (2015) Lateral meningocele with asymmetric canal stenosis: A case study. Saudi J Biol Sci 22: 102-105.

4. Nakasu Y, Minouchi K, Hatsuda N, Nakasu S, Handa J (1991) Thoracic meningocele in neurofibromatosis: CT and MR findings. J Comput Assist Tomogr 15: 1062-1064

5. Wang MY, Levi AD, Green BA (2003) Intradural spinal arachnoid cysts in adults. Surg Neurol 60: 49-55.

6. Kim YJ, Cho HM, Yoon CS, Lee CK, Lee TY, et al. (2011) Surgical treatment of thoracic menigocele associated with neurofibromatosis and kyphoscoliosis. Korean J Thorac Cardiovasc Surg 44: 383-386. 